methionine synthase and the enzyme inhibition is irreversible. (Selzer RR, Rosenblatt DS, Laxova R, Hogan K. Adverse effect of nitrous oxide in a child with 5,10methylenetetrahydrofolate reductase deficiency. N Engl J Med July 3, 2003;349:45-50). (Reprints: Dr Kirk Hogan, Department of Anesthesiology, B6/319 Clinical Sciences Center, 600 Highland Ave, Madison, WI 53792).

COMMENT. Nitrous oxide irreversibly oxidizes the cobalt atom of vitamin $\mathrm{B}_{12}$, and inhibits the activity of methionine synthase which catalyzes the remethylation of 5methyltetrahydrofolate and homocysteine to tetrahydrofolate and methionine. Activated methionine is the principal substrate for the assembly of the myelin sheath, for methyl substitutions in neurotransmitters, and DNA synthesis in tissues. (Chiang PK et al. 1996). The authors propose that a nitrous oxide-induced defect of methionine synthase coupled with the inherited defect of MTHFR were responsible for the patient's death. They cite two recent case reports of infants with acute neurologic deterioration within days of nitrous oxide anesthesia. Both children had severe dietary cobalamin deficiency. The episodes were nonlethal. The above case ending in death followed two nitrous oxide anesthesias a few days apart, one for the biopsy and the other for tumor resection. In addition, the patient had an inborn error of metabolism involving MTHFR. Patients with MTHFR deficiency should not receive nitrous oxide anesthesia.

\title{
APOLIPOPROTEIN C-II DEFICIENCY WITH ENCEPHALOPATHY
}

Two siblings with severe hyperchylomicronemia and encephalopathy in early infancy are reported from Starship Children's Hospital, Aukland, New Zealand. Case 1 presented at 5 weeks of age with tachypnea, feeding problems, and lethargy, which followed a viral upper respiratory tract illness. Parents were second cousins, and some family members had died suddenly in infancy of unexplained illness. Abnormal physical signs included hypotonia, macrocephaly, a white retina, and hepatosplenomegaly. Venous blood was grossly hyperlipemic and appeared "strawberry cream" in color. Triglycerides and cholesterol were grossly elevated, secondary to lipoprotein lipase deficiency. After omitting breast milk and substituting a medium chain triglyceride formula (Monogen), the plasma triglyceride level fell slowly toward normal. MRI showed lipid deposition throughout the brain. Despite correction of lipid blood levels, she had severe developmental delay at 18 month follow-up, and the MRI was unchanged. Plasma apolipoprotein C-II, the lipoprotein responsible for activation of lipoprotein lipase, was absent in the patient and a sibling, also affected (Patient 2), and was below reference levels in all other family members. The sibling who was treated early developed normally. Both patients had a novel homozygous point mutation on sequencing of the APO-C-II gene. The parents and all other siblings were heterozygotes. (Wilson CJ, Oliva CP, Maggi $\mathrm{F}$ et al. Apolipoprotein C-II deficiency presenting as a lipid encephalopathy in infancy. Ann Neurol June 2003;53:807-810). (Dr Wilson, Metabolic Service, Starship Children's Hospital, Auckland, New Zealand).

COMMENT. This rare infantile "lipid encephalopathy" due to apolipoprotein C-II deficiency, and characterized by severe psychomotor retardation, pink venous blood, 
macrocephaly, a white retina (lipid retinalis), and hepatosplenomegaly, may be amenable to treatment with a low fat diet that corrects the hyperchylomicronemia.

\section{MEGALENCEPHALIC CYSTIC LEUKOENCEPHALOPATHY}

A 41 year-old Japanese male diagnosed with megalencephalic leukoencephalopathy (MLC) with subcortical cysts (van der Knaap disease), presenting at 1 year of age with macrocephaly, slowly progressing after 5 years, seizures beginning at 11 years, completely bed ridden at 18 years, and with long survival, is reported from Tokyo Metropolitan Higashiyamato Medical Center for the Severely Disabled, Japan. His motor function had severely deteriorated and his cognitive function was at a 2 year level. MRI revealed marked cerebral atrophy, ventricular enlargement, and large cysts in frontoparietal and anterior temporal areas. A homozygous missense mutation was detected in the MLCl gene. (Saijo H, Nakayama H, Ezoe T et al. A case of megalencephalic leukoencephalopathy with subcortical cysts (van der Knaap disease): molecular genetic study. Brain Dev August 2003;25:362-366). (Respond: Dr Harumi Saijo, Tokyo Metroplitan Medical Center, 3-44-10 Sakuragaoka, Higashiyamato, Tokyo 207-0022, Japan).

COMMENT. Van der Knaap and colleagues described an infantile onset leukoencephalopathy with swelling and a discrepantly mild clinical course in 8 children in 1995. Mutations of MLC1 gene were identified as a cause of the leukoencephalopathy with subcortical cysts in 2001 (Leegwater et al). The changes in size of basal ganglia and diffuse white matter abnormalities that characterize Canavan and Alexander diseases are not evident in MLC.

\section{ACUTE NECROTIZING ENCEPHALOPATHY}

A 14-month-old girl who presented with sudden onset of unreponsiveness following a fever for 2 days developed decerebrate posturing within 9 hours after admission to Children's Memorial Hospital, Chicago, IL. She had no response to deep pain, her reflexes were exaggerated, and plantar responses were extensor. EEG showed slowing but no epileptiform discharges. B!ood counts and chemistries were ncrmal, except for elevated transaminases, with an aspartate aminotransferase level of $190 \mathrm{IU} / \mathrm{L}$ (normal, 22-59 IU/L). CSF analysis was normal. Initial head CT scan was normal, but 10 hours later, a second CT showed new bilateral thalamic hypodensities. MRI with MR angiography and venography, FLAIR imaging, and apparent diffusion coefficient (ADC) mapping confirmed thalamic and periventricular white matter involvement. Infectious, vascular, metabolic, and other etiologies were excluded. When discharged two weeks later to a rehabilitation facility with a diagnosis of acute necrotizing encephalopathy of childhood (ANEC), the child was blind, she responded only to noxious stimuli, and had diffuse spasticity. (Bassuk AG, Burrowes DM, McRae W. Acute necrotizing encephalopathy of childhood with radiographic progression over 10 hours. Neurology 13 May 2003;60:1552-1553). (Reprints: Dr Alexander G Bassuk, Children's Memorial Hospital, Division of Neurology, Box 51, 2300 Children's Plaza, Chicago, IL 60614). 